\title{
EL DESARROLLO ECONÓMICO Y EL CRECIMIENTO DE LA POBLACIÓN *
}

\author{
VÍctor L. URQUIDI \\ El Colegio de México
}

DuRANTE LARgos lustros, los economistas no se ocuparon del incremento y el cambio demográficos sino como datos que había que acomodar en alguna forma a la teoría del desarrollo o a la de la determinación del ingreso. No percibían adecuadamente la integración del elemento población en los procesos sociales dinámicos, en los procesos económicos y, en particular, en los cambios de estructura que implica el desarrollo. Mientras el crecimiento demográfico fue lento y no se registraron los agudos movimientos migratorios entre las áreas rurales y las urbanas que son hoy característicos del proceso de desarrollo, tal vez se justificara, desde el punto de vista de los economistas, esa relativa desatención a la demografía. No se carecía de antecedentes sobre el fenómeno de la población, desde las preocupaciones malthusianas hasta las teorías del óptimo de la población en boga hace unos cuarenta años. Por otro lado, las grandes migraciones internacionales del siglo XIX entre Europa y el continente americano, y otras no insignificantes entre el Lejano Oriente y determinadas áreas de este continente, debieran haber hecho pensar en su significación. Pero fue todavía en parte la era de las grandes expansiones del siglo XIX, cuando acudían capital y gente a la apertura de nuevas tierras, así como a la explotación de productos básicos ávidamente solicitados por las naciones industriales en cierne, primero Europa y después Estados Unidos y Canadá. El sentir general era el de que sobraba tierra y faltaba población; ésta llegó, por cierto, junto con capital suficiente para trabajar la tierra, abrir las minas y poner en actividad nuevas fuentes de bienes materiales.

Hoy día nos asalta claramente la duda de que la situación es radicalmente distinta. No es tan obvia como antes la posibilidad de abrir a la actividad económica grandes extensiones de nuevas tierras, al menos sin dotaciones de capital fuertes y todavía inconmesurables y sin grandes programas previos de saneamiento y acondicionamiento. Por otro lado, la gran expansión económica de los países latinoamericanos se ha dejado de caracterizar, como lo fue hasta hace unos 30 a 40 años, como una respuesta a la demanda de los países industriales que se ejercía respecto a crecientes necesidades de materias primas y productos alimenticios. Ha habido cambios sustanciales en la estructura económica, producto de la adaptación de las políticas económicas

* Ponencia presentada al Segundo Coloquio sobre Población y Desarrollo, del Population Reference Bureau, en Barranquitas, Puerto Rico, diciembre 14-16, 1968. 
y sociales a las modalidades impuestas por el crecimiento interno. Por la imposibilidad de financiar las importaciones adecuadamente con las divisas obtenidas con la exportación, se ha dado un fuerte impulso, en los últimos años, a la industrialización, la que requiere, en gran parte, estar concentrada en los centros urbanos, provistos de todos los servicios técnicos y financieros. El atractivo de la vida urbana, la improductividad milenaria de muchas zonas agrarias y la relativa desatención a la modernización de la agricultura, han alentado considerablemente la migración interna hacia las ciudades de todos los tamaños. La expansión de la educación y los servicios de salud, centrados predominantemente también en las ciudades, han sido factor de atracción de la población a éstas, a la vez que han influido de manera marcada en las variables demográficas básicas.

Nada quizá tan notable como los cambios ocurridos en la mortalidad de los latinoamericanos, que no tienen precedente, por su intensidad, en la historia de la humanidad. El fuerte descenso de la mortalidad ha sido decisivo en el crecimiento de la población latinoamericana de unos 60 millones a principios de siglo a más de $260 \mathrm{mi}$ llones en la actualidad. El contraste con los países hoy desarrollados se ilustra con las cifras, tantas veces citadas, de Inglaterra y Gales, por un lado, y Costa Rica, Chile, México o Venezuela, por otro. A Inglaterra le llevó 150 años reducir a la mitad la tasa bruta de mortalidad que manifestaba a mediados del siglo xvirI (33 por millar), mientras que Costa Rica ha logrado esa misma proporción de descenso en tan sólo 20 años, en el segundo tercio del actual siglo; período en el cual países como Chile, México, Venezuela, El Salvador y otros han obtenido reducciones de 35 a $45 \%$, con posibilidades de abatir aún más la mortalidad.

Una de las características de los cambios en la mortalidad es que su reducción en los últimos decenios ha ocurrido principalmente en los grupos de la población de edades jóvenes. Para dar un ejemplo, en México, entre 1930 y 1960, la mortalidad infantil se redujo en más del $50 \%$, pero la proporción fue mayor, del $75 \%$, en las edades de 1 a 14 años; y también, del $60 \%$, en las edades de 15 a 30 años. Estas disminuciones de la mortalidad, aparte de su efecto sobre la esperanza media de vida, significan un rejuvenecimiento de la población al aumentar la proporción de personas de edad temprana; pero también resultan en un incremento de la fecundidad por aumento del número de mujeres que alcanzan a entrar en la edad fecunda, así como por ampliación de su período de procreación.

Así, el fenómeno de la menor mortalidad, deseable desde todos los ángulos, sociales y humanos, es a su vez factor del incremento de la fecundidad, y la expansión de los servicios de salud e higiene, y el mayor alcance de la educación general, actúan doblemente sobre la mortalidad y sobre la fecundidad. Con el tiempo, esta última se ha elevado en América Latina en algunos de los países más poblados, contrariamente a lo que ha ocurrido en la historia de los países hoy industrializados, donde el crecimiento económico y la educación llevaron a una disminución de la tasa de natalidad. En el período 19601965, ocho países latinoamericanos han registrado tasas de natalidad entre 40 y 44 por millar, otros siete países, tasas de 45 a 49 por millar, 
y un país ha alcanzado el nivel extraordinario en exceso de 50 al millar. Es sabido que sólo dos países muestran una natalidad inferior a 30 al millar, que es todavía superior a lo que ocurre en la mayoría de los países industrializados. Parece percibirse en algunos países latinoamericanos un principio de descenso de la natalidad, o al menos su estabilización, pero todavía a tasas muy elevadas que, por lo demás, tampoco tienen precedente en los períodos de gran expansión económica de las naciones hoy desarrolladas de Europa y América del Norte.

La combinación de una mortalidad en descenso con una fecundidad alta y estabilizada, además de producir tasas de incremento elevadas y crecientes, que en el promedio latinoamericano son ya de $2.9 \%$, pero que en varios países exceden del $3 \%$ y en algunos del $3.5 \%$, genera también estructuras por edades que elevan la tasa de dependencia tanto para la familia como para la economía en su conjunto. Cada adulto económicamente activo tiene que proveer con su trabajo a las necesidades de una proporción mayor, y en muchos casos creciente, de personas que están en la niñez o en la adolescencia, lo que a su vez significa una carga mayor para los servicios de educación, de salud y bienestar y otros. A falta de una expansión adecuada de la educación, el incremento de la proporción de adolescentes presiona sobre el mercado de trabajo y contribuye a la condición de subocupación y desocupación también tan característica de los países subdesarrollados, entre ellos la mayoría de los latinoamericanos.

El rejuvenecimiento de la población se refleja además en algunos rasgos importantes de la fuerza de trabajo y en su dinámica; en la actualidad, y por muchos años adelante, casi la totalidad de las personas que esperen entrar a la fuerza de trabajo tendrán edades inferiores a los 20 años. En países con estructura similar a la de la población de México, por ejemplo, el $94 \%$ de la población masculina urbana y rural entra a la actividad económica antes de los 20 años, y en el caso de la femenina, el $98 \%$ tratándose de la urbana y algo menos en la rural. Pero es de notar que el $58 \%$ de la población urbana ingresa a la actividad económica de hecho antes de los 15 años de edad, y que la edad media de incorporación al trabajo es de 15 años. Sin embargo, las tasas globales de participación son bajas e incluso muestran tendencia a disminuir. En México, nuevamente, la llamada tasa bruta de actividad en las äreas urbanas se redujo de 31.0 a $29.7 \%$ entre 1960 y 1965 , y en las áreas rurales, de 29.1 a $28.9 \%$ en el mismo período. Estas reducidas tasas, que contrastan con las de 40 a $45 \%$ y más en los países industrializados, reflejan la estructura por edades, así como la insuficiente absorción de población en el trabajo urbano. ${ }^{1}$

A este respecto, cabe hacer notar que si bien se ha determinado que en los países latinoamericanos de mayor incremento demográfico la fecundidad urbana es inferior a la rural, la diferencia no es muy grande, y no tiende a ampliarse por mayor descenso de la fecundidad urbana debido a la naturaleza de la fuerte migración entre las áreas

1 José B. Morelos, "Entradas a la actividad, salidas y vida media activa en México, 1960-1965”, Demografía y Economía, Vol. II, Núm. 1, 1968, pp. 20-27. 
rurales y las urbanas, que lleva al medio urbano los factores sociales familiares que determinan una fecundidad muy elevada.

Pero, por otro lado, no obstante la tasa de migración rural-urbana, que se expresa en crecimientos de ciudades del orden del 5, el 7 v el 10 y más por ciento anual (incluido el incremento natural en las mismas), los países latinoamericanos continúan siendo, en su mayoría, países de características predominantemente rurales. En 1960, se estimaba que los dos tercios de la población de América Latina vivía en lugares de menos de 20000 habitantes, que son centros comerciales y agropecuarios más que entidades industrializadas o en principios de desarrollo industrial. A esto hay que agregar, en algunos países, como México, una enorme dispersión demográfica en pequeños poblados y lugares apartados. La población rural de América Latina pesa sobre estructuras agrarias que, en general, reflejan una gran concentración de la propiedad, pero aun en los pocos países que han llevado a cabo reformas importantes al sistema de tenencia de la tierra la dimensión de la parcela media es tan pequeña que empieza a advertirse un grave problema de sobrepoblación rural, sobre todo en las áreas de baja productividad y escasa comercialización de la agricultura.

El fenómeno del crecimiento urbano apenas si ha aliviado el problema rural en parte, a la vez que ha hecho surgir otros problemas derivados de la falta de capacidad de los sistemas industriales y urbanos para absorber el incremento rápido de la población que se establece en las ciudades. Éste es otro fenómeno totalmente distinto al de los países industrializados. Lo común en los países latinoamericanos de mayor dinamismo demográfico es que la proporción de población urbana (en ciudades de 20000 y más habitantes) se duplique en 20 a 25 años, mientras que este proceso tardaba de 35 a 45 años en Estados Unidos o Inglaterra en épocas de gran expansión industrial. La urbanización rápida, como es bien sabido, ha ejercido graves presiones sobre los programas de vivienda y de mejoramiento urbano, así como sobre los sistemas educativos y de servicios sociales.

$\mathrm{La}$ anterior caracterización de algunos de los aspectos salientes de la problemática demográfica de América Latina sugiere claramente que nuestros países se encuentran en un proceso de cambio totalmente nuevo, sin precedente en la experiencia económica, social, administrativa o política. Se trata de un incremento demográfico muy rápido y sostenido, poco susceptible de modificación en un plazo razonable y que imprimirá un sello marcado a las formas de vida y de convivencia de las próximas dos generaciones. De seguir las tendencias actuales, se prevé una población latinoamericana de cerca de $275 \mathrm{mi}$ llones para 1970, 365 millones para 1980 y alrededor de 650 para el año 2000.

Independientemente de cualquier consideración social, política o ética, cabe preguntarse si semejante dinámica demográfica afectará apreciablemente, de manera favorable o negativa, el desarrollo económico de la región.

$\mathrm{Al}$ abordar este tema, es pertinente hacer hincapié en que ha habido una tendencia a simplificar con exceso el problema, y en particular a presentar disyuntivas que no son reales. Por ejemplo, se han hecho afirmaciones en el sentido de que el incremento demográfico ejerce 
una influencia netamente negativa sobre el crecimiento económico, por lo general a base de comparar la tasa de aumento de la población con la del producto bruto o la de la producción de alimentos; y de ello se han sacado conclusiones demasiado ligeras en el sentido de que una reducción de la natalidad implica automáticamente una elevación del nivel del producto bruto por habitante, o del suministro de alimentos.

En el otro extremo, se han hecho afirmaciones que rayan en lo irracional según las cuales el vasto territorio de la América Latina o de algunos de sus países tiene cabida para números ilimitados de habitantes, como si el fin último de la actividad social fuera simplemente llenar kilómetros y más kilómetros cuadrados de más y más gente.

Ninguna de estas posiciones extremas contribuye mucho a esclarecer la naturaleza verdadera de los problemas de relación entre población y desarrollo, que sólo pueden comprenderse a base de considerar los muchos elementos que componen el conjunto, sus interrelaciones y la dinámica de los cambios de estructura.

Pero aun al mayor nivel de agregación cabe preguntar si el desarrollo económico latinoamericano ha sido tan rápido y pleno de éxito en los años recientes de mayor incremento demográfico como para concluir que el primero se puede impulsar satisfactoriamente en forma independiente de lo que suceda con la población. El interés que ponen los gobiernos latinoamericanos, y con ellos los diferentes sectores de la colectividad, en acelerar el crecimiento económico revela que, en principio, la tasa de crecimiento de la actividad económica dista mucho de satisfacer las necesidades actuales y menos aún las aspiraciones de los pueblos latinoamericanos. Por desgracia, la historia reciente, que ya va alargándose a dos decenios, no indica el dinamismo económico deseado. Los problemas del crecimiento económico son verdaderamente agudos. Éstos son problemas viejos, pero a la vez nuevos, porque apenas muy lentamente han cambiado las actitudes hacia ellos y se han podido reconocer y esbozar lineamientos convenientes de desenvolvimiento.

Debido a factores de orden externo, relacionados con la insuficiente expansión de los mercados de los productos latinoamericanos, pero también a obstáculos y deficiencias internos, el crecimiento económico general de América Latina se ha debilitado a partir de 1950. Según las cifras elaboradas por la CEPAL, el producto bruto por habitante crecía a razón de $2.2 \%$ al año entre 1950 y 1955 . A esa tasa se requerían 32 años para duplicar el producto per capita, representativo en términos aproximados del nivel medio de vida. Pero entre 1955 y 1960 , la tasa de incremento fue de 1.8 , y entre 1960 y 1965 de $1.7 \%$ anual. A estas últimas tasas el período requerido para duplicar el nivel de vida es de 41 a 42 años. Finalmente, en 1966 y 1967, el promedio de incremento fue de $1.3 \%$ anual, con lo que excede de 50 años el período necesario para duplicar el producto per capita. ${ }^{2}$ Estas tasas de crecimiento contrastan con las logradas en países de Europa occidental y oriental, que son del orden de 5 a $8 \%$ anual por habitante (lo que

2 CEPAL, Estudio económico de América Latina, 1967. Nueva York, Naciones Unidas, Publ. S.69.II.G.1, 1968, p. 3, cuadro 1. 
implica duplicación del nivel medio de vida en 15 y hasta en 9 años); contrastan igualmente con las obtenidas en Israel, Japón y otros países. Debe tenerse en cuenta que la situación en América Latina no es pareja, pero aun países como México, Brasil, Venezuela o Nicaragua, que durante algunos años han logrado incrementos de 3 a $5 \%$ por habitante, tardarían de 15 a 24 años en multiplicar por dos su producto per capita.

Lo anterior no quiere decir que si la tasa de incremento demográfico fuera menor, por ejemplo, del $2.5 \%$ en lugar del $3.0 \%$, se habría obtenido por ese solo hecho un mejoramiento mayor del nivel de vida, ni que el decaimiento de la tasa de aumento del producto per capita se habría detenido. Pero tampoco quiere decir que con un aceleramiento del ritmo del crecimiento del producto, como resultado de un esfuerzo de inversión y coordinación de la política económica hasta ahora desconocidos, cuyo objetivo fuera siquiera igualar las metas impuestas en la Alianza para el Progreso, se habría obtenido un alza apreciable del nivel medio de vida.

Es decir, la respuesta al problema demográfico que se plantea en América Latina no está simplemente en decir que la solución la da el desarrollo económico, primero porque tendría éste que ser varias veces superior al ritmo que acusa actualmente, y en segundo lugar porque la experiencia y las perspectivas en que se desenvuelve América Latina no autorizan a prever que las tasas globales de desarrollo sean adecuadas ni puedan serlo en el futuro inmediato.

Luego existe un argumento de orden general a favor de una política de población que, entre otras cosas, considere la posibilidad de una reducción de la tasa de fecundidad, no como solución, sino como alivio al grave problema del desarrollo económico y social. La tasa de mortalidad tiene probabilidades de seguir disminuyendo moderadamente, pero a sus niveles actuales no es ya el factor determinante del elevado incremento demográfico. La única variable sobre la que cabe actuar, por efectos de la educación y la urbanización, con los cambios de actitudes que esto envuelve, y por medio de programas de planificación familiar, es la fecundidad, tanto la rural como la urbana.

En la Conferencia sobre Población y Desarrollo Económico celebrada en Caracas en 1967, bajo los auspicios de la Organización de los Estados Americanos y la Oficina Panamericana de la Salud y otras organizaciones, se hizo especial hincapié en la complejidad de las relaciones entre población y desarrollo. ${ }^{3}$ En particular, el problema se desagregó en varios de sus elementos y se evidenció el distinto efecto que el incremento demográfico tiene al nivel global y al nivel sectorial, y en relación con problemas de educación, de salud, de vivienda, de desequilibrio regional y de empleo, así como los que en especial tiene al nivel familiar entre las poblaciones llamadas marginales. Cabe advertir que todos estos fenómenos presentan en la mayoría de los países latinoamericanos caracteres agudos. Un reciente informe de la CEPAL en que se examina la situación social de América Latina

3 Véase "Declaración y recomendaciones de la Reunión sobre Políticas de Población en Relación al Desarrollo de América Latina", reproducido en DEMoGRAFÍA Y ECONOMfa, Vol. I, Núm. 3, 1967, pp. 408-428. 
afirma que "es evidente un clima de insatisfacción generalizada respecto a lo logrado desde comienzos de la presente década y de incertidumbre sobre lo futuro".4 Existe rigidez estructural y los estratos marginales no se han beneficiado gran cosa. Se estima que el $25 \%$ de la población urbana de América Latina vive en los llamados asentamientos irregulares, y que este estrato de la población se incrementa a razón de 10 a $15 \%$ anual. La industrialización no ha resuelto los problemas de empleo, y de hecho se ha reducido la proporción de población activa que trabaja en la industria, mientras se ha elevado la que encuentra ocupación en los servicios. Por añadidura, existe, además del subempleo muy extendido, el peligro de una desocupación abierta. Todo ello dentro del marco de una situación en que el $11 \%$ de la población activa, que genera un $40 \%$ del producto global latinoamericano, tiene niveles de productividad cercanos a los de Europa occidental, mientras un $40 \%$ alcanza apenas los niveles de los países más pobres de Asia. ${ }^{5}$

Se reconoce claramente en todos los estudios recientes, tanto de las Naciones Unidas, la UNESCO y otras entidades de este sector, como del Banco Interamericano de Desarrollo y las demás entidades interamericanas, que por más que ha habido una expansión sin precedente de los programas educativos, de la salud y de vivienda, así como de seguridad social en los últimos años, todos ellos distan mucho de ser satisfactorios y en algunos casos apenas si se acercan medianamente al ritmo de aumento de las necesidades. América Latina se encuentra ante el problema sin antecedente histórico de tener que hacer frente a masivos programas de bienestar social al mismo tiempo que debe destinar las mayores proporciones posibles de recursos reales a la ampliación de su infraestructura económica, al aumento de la capacidad de producción industrial, al mejoramiento de la productividad agrícola, al fortalecimiento de sus instituciones financieras, al incremento de la exportación y a todos los demás elementos que, en su conjunto, constituyen una política apetecible de desarrollo económico a la vez que social.

El incremento demográfico demasiado rápido, que evidentemente dificulta la solución simultánea de todos estos problemas, afecta incluso algunas de las variables económicas fundamentales. La inversión bruta en América Latina constituye en promedio del 16 al $18 \%$ del producto bruto (aun cuando en algunos países es desde luego superior). Esta proporción no es en verdad muy elevada, si se compara con la que han logrado algunas de las naciones, como Japón, que han hecho esfuerzos espectaculares de desarrollo en los últimos veinte años. No sólo no es muy elevada, sino que, conforme a datos recientes de la CEPAL, ha venido declinando ligeramente. ${ }^{6}$ Puede afirmarse que dicho coeficiente de inversión bruta, cualquiera que sea la

4 CEPAL, op. cit., Cap. II, p. 32.

5 Ibid., pp. 45, 47 y passim. En algunos países, la desocupación urbana alcanza proporciones muy elevadas; por ejemplo, en Buenos Aires $(6.5 \%$ de la población activa en abril de 1966); en Uruguay (8.5\%); en Santiago de Chile (de 4.3 a $7.6 \%$ entre 1961 y 1966); en Venezuela, la República Dominicana (17.8\%) y Panamá (de 12 a $17 \%$ ). Véanse las pp. 45-46 del Estudio citado.

6 Ibid., p. 5, cuadro 4 . 
tasa de incremento demográfico positiva de un país, es insuficiente para asegurar un cambio estructural de importancia y la puesta en marcha de un proceso de desarrollo firme y sostenido.

Se dice mucho acerca de si el desarrollo sería más eficiente si los métodos de producción implicaran menor uso relativo de capital y mayor empleo relativo de mano de obra. Lógicamente, así parecería a primera vista en los países en donde lo que abunda es la mano de obra barata y escasea el capital real. Pero las corrientes tecnológicas modernas, nacidas en su mayor parte en los países más adelantados donde las relaciones entre trabajo y capital son las contrarias, tienden a producir economía de mano de obra; y estas corrientes son irreversibles, particularmente en la industria y la agricultura modernas, de tal suerte que tiende a emplearse más capital por persona ocupada y tiende a sustituirse mano de obra por equipo. Luego, el esfuerzo que habrá que hacer en materia de inversión real para dotar a la población activa del capital por persona necesario para los incrementos tradicionales de la producción por habitante tendrá que ser cada vez más intenso. A lo que habría que agregar la dotación adicional de capital para hacer más rápido el incremento de la productividad. Y sería preciso agregar, todavía más, las dotaciones de capital requeridas para absorber en la población activa aquella parte de la fuerza de trabajo que se encuentra desocupada, los incrementos de ésta resultantes del aumento demográfico y el rejuvenecimiento de la población, y los aumentos potenciales efectivos provenientes de la transformación deseable de subocupados en trabajadores plenamente ocupados.

Estas consideraciones sobre el efecto del incremento demográfico en las necesidades de inversión deben adicionarse con otras más, referentes al hecho de que a medida que se eleva la proporción de población joven, dependiente de la que trabaja, la colectividad se ve precisada -o por lo menos así debería ocurrir - a destinar a inversiones no directas ni inmediatamente productivas, tales como educación, salud, bienestar, mejoramiento urbano, vivienda, etc., proporciones crecientes de recursos. Es fácil apreciar, en consecuencia, cómo una menor tasa de incremento de la población contribuiría a aliviar el difícil problema de asignar mayores recursos a la inversión en sociedades democráticas liberales en que el consumo tiende a tener prioridad, tanto por el lado del sector público como por el del sector privado, sobre todo el de ingresos medianos y altos determinado por la estructura social prevaleciente en América Latina.

El aumento de la población actúa también en la capacidad de financiamiento de la inversión. La apenas moderada tasa de inversión de los países latinoamericanos tiene su contrapartida en el insuficiente ahorro, lo mismo personal, de las empresas o del propio sector público. En términos generales, la capacidad de ahorro de una economía está determinada por la estructura social y de la propiedad, por el grado de avance tecnológico y su impacto en la productividad y por la distribución del ingreso, conjuntamente con la naturaleza de la política fiscal. El incremento rápido de la población, con su rejuvenecimiento consiguiente, tiende a favorecer el consumo a expensas del ahorro, lo mismo el consumo familiar que el que lleva a cabo el sector 
público para proveer los servicios que la población demanda. Diversos estudios han demostrado que a cualquier nivel de producto bruto, la formación de ahorros se reduce bajo la influencia de tasas de natalidad muy elevadas, y éste es el caso de América Latina en lo general.? En consecuencia, es éste otro aspecto en que puede percibirse que una política de población tendiente a frenar la tasa de incremento demográfico favorecería el robustecimiento de la tasa global de ahorro interno, y en esa medida, aparte de otros efectos, reduciría la dependencia excesiva del capital del exterior.

El examen de estos problemas gruesos de desarrollo económico lleva a la conclusión de que representaría cierto alivio en su solución una menor tasa de natalidad, por cualquier medio que se produzca ésta. De ninguna manera se eliminaría la dificultad de llevar a cabo un proceso racional y dinámico de desarrollo, como tampoco se eliminaría el problema demográfico en sí. Siempre habrá tales problemas, entre otras cosas porque los acomodos y adaptaciones no se producen automáticamente, y porque, como ya es el caso, subsistirán fuertes desequilibrios regionales en el interior de los países de América Latina y entre las áreas rurales y las urbanas. Habrá sobrepoblación, si no global, cuando menos en relación con los recursos económicos y financieros y en algunos casos en relación con los físicos. Habrá en muchos casos sobrepoblación rural. Habrá por lo menos sobrepoblación regional. Y habrá seguramente sobrepoblación urbana.

Los factores condicionantes del desarrollo latinoamericano podrían variar imprevisiblemente, aun cuando no es probable que se aprecie, dada la experiencia del último decenio, ningún cambio radical. En todo caso, serán distintos para cada país, y no podrá compararse la situación, por ejemplo, de El Salvador o Haití, con la de Brasil o Argentina. Pero aun estos últimos dos países, de gran territorio y enormes recursos no explotados, padecen subdesarrollo, subempleo, excesiva concentración urbana, rezago en los programas sociales, y en general, muchas de las características de un esfuerzo insuficiente de desenvolvimiento, cualquiera que sea su dinámica demográfica actual. Lo determinante es y seguirá siendo la disponibilidad de capital, la capacidad para utilizarlo racionalmente y organizar la producción, la viabilidad de sistemas económicos dinámicos y fluidos y la capacidad de planeación, es decir, de previsión de necesidades y de preparación para hacer frente a ellas. Lo que es un problema político y administrativo, además de económico y sociológico.

Como conclusión general, puede adelantarse que una política tendiente a reducir la tasa de incremento de la población tendría en la mayoría de los países latinoamericanos un efecto benéfico en los esfuerzos de desarrollo económico y social: a corto plazo, vía el ingreso familiar de las poblaciones marginales, las rurales y las de salario bajo; a plazo mediano y largo, vía los programas de inversión social y los de desarrollo de la infraestructura económica, los de absorción

7 Véase la ponencia presentada por el autor al Congreso Mundial de Población, Belgrado, 1965, "El crecimiento demográfico y el desarrollo económico latinoamericano", Demograffa y Economfa, Vol. I, Núm. 1, 1967, pp. 1-8, y las referencias en ella proporcionadas. 
de fuerza de trabajo en la actividad industrial y los servicios, los de desarrollo regional interno y los de gastos corrientes.

El crecimiento demográfico rápido y el aceleramiento del desarrollo económico son dos fenómenos sociales a los que América Latina se enfrenta con poca o ninguna experiencia, con poca o ninguna teoría y apremiada por la urgencia de hacer una labor efectiva que redunde en bienestar para la población y capacidad para ajustarse al cambio y sentar las bases de una evolución adecuada a largo plazo. Estos dos fenómenos ocurren en una época en que se advierte una transición en las ideas. Se pasa, tal vez con creciente velocidad, de una época en que se aceptaba la inevitabilidad de los hechos sociales a otra en que se sienten la necesidad de influir en ellos y de orientarlos conforme a objetivos determinados. En materia de planeación económica, se admite ya esta necesidad, aun cuando débilmente; pero en materia de planeación social, la conciencia de esa necesidad es muy baja. Priva en las mentes todavía un liberalismo económico que conduce al peligroso espejismo de que con sólo un poco de planeación económica y social pero a la vez con pleno determinismo demográfico se puede alcanzar un objetivo de bienestar acorde con las aspiraciones populares. Es urgente que el problema demográfico se integre en el conjunto de los demás problemas que aquejan al devenir económico y social de América Latina. 